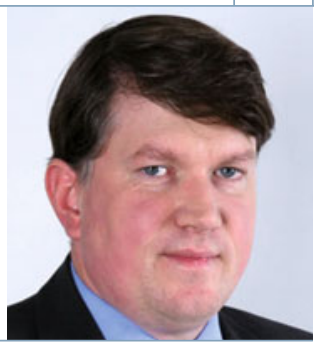

\title{
Klare gesetzliche Vorgaben für eine zukunftsfähige Biotechnologie!
}

10.1007/s12268-014-0467-5

(C) Springer-Verlag 2014

- Forschen Sie, womöglich an einer öffentlichen Hochschule? Dann sind Sie mit dem Gentechnikrecht vertraut und bilden Ihre Studierenden und Doktoranden entsprechend aus. Sie schreiben Anträge für Fördermittel, die je nach Bundesland berücksichtigen, dass Gentechnik nicht gefördert wird, und werben um Kooperationsprojekte mit Unternehmen (wie dies auch von nationalen und EU-Förderern gewünscht wird). Je nach Bundesland müssen Sie Ihre Forschungspartner aus der Wirtschaft davon überzeugen, dass Thema und Umfang der Kooperation veröffentlicht werden. Geht es nach dem deutschen Ethikrat, müssen Sie außerdem demnächst überlegen, ob Ihre Forschungsergebnisse missbraucht werden könnten - und zwar nicht nur nach den heute geltenden Dual-Use-Kriterien, sondern auch darüber hinaus. Vielleicht müssen Sie Ihr Forschungsvorhaben zukünftig auch im Rahmen eines Bürgerdialogs vorstellen und Zustimmung dafür einwerben...

Damit wir uns nicht falsch verstehen: Gesellschaftlicher Diskurs darüber, was wir forschen und vor allem anwenden wollen, ist ebenso notwendig wie strenge Sicherheitsregeln und Risikoabwägungen. Auch hat der Steuerzahler ein Recht darauf zu erfahren, was mit seinen Geldern an öffentlichen Einrichtungen passiert. Diese berechtigten Anliegen dürfen aber nicht dazu führen, dass Gesetze, Richtlinien, Regeln und Begleitprozesse der Forschung die Luft abschnüren. Mit dem Gentechnikrecht liegt ein gesetzlicher Rahmen vor, der gewährleistet, dass der Umgang mit gentechnisch veränderten Organismen nur mit Sachkunde unter entsprechend hohen, abgestuften Sicherheitsregeln erfolgt. Derzeit ist aber zu beobachten, dass eine Vielzahl zusätzlicher Anforderungen diskutiert wird, die diesen Rahmen immer enger stecken. Das reicht von immer weiterführenden Transparenzregelungen für öffentliche Einrichtungen, die Kooperationen zwischen Hochschulen und Industrie verkomplizieren, bis zur Einrichtung

\footnotetext{
1 Die vollständige Studie „Biotechnologie - der Schlüssel zur Bioökonomie“ können Sie unter www.dechema.de/studien.html kostenlos herunterladen.
}

einer Ethikkommission, die über die Freigabe einer Forschung im Sinne der Dual-Use-Problematik entscheiden soll. Schlimmer noch: Diese Vorschläge kommen aus den unterschiedlichsten Richtungen, scheinen untereinander wenig abgestimmt und fallen je nach Bundesland höchst unterschiedlich aus.

Gleichzeitig fließen - erfreulicherweise! erhebliche Beträge sowohl auf nationaler wie auf EU-Ebene in die biotechnologische Forschung, und die Biotechnologie wird regelmäBig als eine Schlüsseltechnologie für die Bioökonomie und für den medizinischen Fortschritt bezeichnet. Wir müssen aufpassen, dass wir nicht auf der einen Seite Geld in notwendige Entwicklungen stecken, die wir auf der anderen Seite durch Überregulierung oder durch Verfahren, die so aufwendig sind, dass sich ihnen niemand aussetzen kann oder will, zunichtemachen. Wir sehen die aktuelle Entwicklung mit großer Sorge. Deutschland ist heute noch mit Ausnahme der Pflanzenbiotechnologie - ein starker Standort der Biotechnologie. Wir brauchen Innovationen aber auch in diesem Gebiet, um unsere Position international zu behaupten.

Welche wertvollen Impulse auch in Zukunft für die gesellschaftliche und wirtschaftliche Entwicklung ausgehen werden, zeigt das Zukunftsforum der DECHEMA eindrucksvoll in der neuen Studie „Biotechnologie - der Schlüssel zur Bioökonomie“" 40 Jahre nach der ersten DECHEMA-Studie zur Biotechnologie haben die Autoren anhand der Themenkreise Gesundheit, Ernährungssicherheit, nachhaltige Agrarproduktion, industrielle Nutzung nachwachsender Rohstoffe und Energieträger aus Biomasse verdeutlicht, dass ohne eine moderne Bio- und Gentechnik die Herausforderungen der Zukunft nicht zu bewältigen sind. Die Autoren stellen fest, dass eine neue Phase der Biotechnologie begonnen hat, die viele Innovationen hervorbringen wird. Neue Technologien ermöglichen es immer besser, biologische Systeme quantitativ zu erfassen, zu verstehen und zu modellieren. Zugleich reifen Methoden heran, um ganze Genome gezielt zu verändern, umzubauen und sogar komplett zu synthetisieren. Damit ist das rationale, zweckgerichtete Design von Zellen möglich geworden. Es verspricht visionäre Lösungen: Biotech-Pflanzen, die auch auf min- derwertigen oder trockenen Böden wertvolle Rohstoffe produzieren, „mikrobielle Chemiefabriken“, die Holz als Nahrungsquelle nutzen, um daraus Chemikalien herzustellen, Algen, die mit der Energie des Sonnenlichts Kohlendioxid und Wasser direkt in Kraftstoffe umwandeln, oder Bakterien, die elektrische Energie dazu nutzen. Diese Designer-Organismen könnten helfen, den Kraftstoffbedarf ressourcenschonend zu decken, ohne wertvolle landwirtschaftliche Flächen zu beanspruchen. Nützlich werden auch neu konstruierte Mikroorganismen werden, die aus rohstoffarmen Erzen und Recyclinglaugen seltene Metalle gewinnen oder Umweltbelastungen mindern. Das Potenzial dieser „Synthetischen Biologie“ ist kaum abschätzbar - insbesondere in der Medizin, wo es um die Möglichkeiten von Gentherapie und Stammzellforschung erweitert werden wird.

Wir müssen die Potenziale der Technologien, die uns zur Verfügung stehen und noch entwickelt werden, verantwortungsvoll nutzen. Dafür muss im vorhandenen gesetzlichen Rahmen Forschung frei von ideologiegetriebener Überregulierung möglich sein.

Anstelle von unabgestimmten und aktionistischen Maßnahmen muss ein offener, sachlicher, faktenbasierter gesellschaftlicher Dialog treten, der in praktikable, klare und einheitliche Regeln mündet. Dafür stellen wir uns jederzeit gerne zur Verfügung!

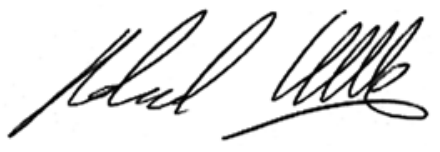

Roland Ulber, Vorsitzender der DECHEMAFachgemeinschaft Biotechnologie im Namen des Vorstands der Fachgemeinschaft

\section{Korrespondenzadresse:}

Prof. Dr. Roland Ulber

Technische Universität Kaiserslautern

Lehrgebiet Bioverfahrenstechnik

Gottlieb-Daimler-Straße

Gebäude 44/519

D-67663 Kaiserslautern

Tel.: 0631-205-4043

Fax: 0631-205-4312

ulber@mv.uni-kl.de 